\title{
Repulsive Casimir Force in Chiral Metamaterials
}

\author{
R. Zhao, ${ }^{1,2}$ J. Zhou, ${ }^{1}$ Th. Koschny, ${ }^{1,3}$ E. N. Economou, ${ }^{3,4}$ and C. M. Soukoulis ${ }^{1,3}$ \\ ${ }^{1}$ Ames Laboratory and Dept. of Phys. and Astronomy, Iowa State University, Ames, Iowa 50011, U.S.A. \\ ${ }^{2}$ Applied Optics Beijing Area Major Laboratory, Department of Physics, Beijing Normal University, Beijing 100875, China \\ ${ }^{3}$ Institute of Electronic Structure and Laser, FORTH, and Department of Materials \\ Science and Technology, University of Crete,71110 Heraklion, Crete, Greece \\ ${ }^{4}$ Department of Computational and Data Sciences, George Mason University, Fairfax, Virginia 22030, USA
}

(Dated: August 30, 2018)

\begin{abstract}
We demonstrate theoretically that one can obtain repulsive Casimir forces and stable nanolevitations by using chiral metamaterials. By extending the Lifshitz theory to treat chiral metamaterials, we find that a repulsive force and a minimum of the interaction energy exist for strong chirality, under realistic frequency dependencies and correct limiting values (for zero and infinite frequencies) of the permittivity, permeability, and chiral coefficients.
\end{abstract}

PACS numbers: 42.50.Ct, 78.20.Ek, 12.20.-m

Following the original Casimir paper [1] for the attraction of two media, 1 and 2 occupying half spaces, $z<0$ and $z>d$, respectively, and such that the electromagnetic fields are confined exclusively in the vacuum region between them, Lifshitz [2] generalized the calculation of this force to the case that these two media are characterized by frequency-dependent dielectric functions $\epsilon_{1}(\omega)$ and $\epsilon_{2}(\omega)$. Subsequently, there was further generalization to general bi-anisotropic media [3]. The formula for the force or the interaction energy per unit area can be expressed in terms of the reflection amplitudes, $r_{j}^{a b}$ $(j=1,2)[4]$, at the vacuum/medium $j$ interface, giving the ratio of the reflected EM wave of polarization $a$ by the incoming wave of polarization $b$. Each $a$ and $b$ stands for either electric (TM or $\mathrm{p}$ ) or magnetic (TE or $\mathrm{s}$ ) waves. The frequency integration is completed along the imaginary axis by setting $\omega=i \xi$. The formula for the interaction energy per unit area becomes [5]

$$
\frac{E(d)}{A}=\frac{\hbar}{2 \pi} \int_{0}^{\infty} d \xi \int \frac{d^{2} \mathbf{k}_{\|}}{(2 \pi)^{2}} \ln \operatorname{det} \mathbf{D},
$$

where $\mathbf{D}=1-\mathbf{R}_{1} \cdot \mathbf{R}_{2} e^{-2 K d}, K=\sqrt{\mathbf{k}_{\|}^{2}+\xi^{2} / c^{2}}$, and

$$
\mathbf{R}_{j}=\left|\begin{array}{cc}
r_{j}^{\mathrm{ss}} & r_{j}^{\mathrm{sp}} \\
r_{j}^{\mathrm{ps}} & r_{j}^{\mathrm{pp}}
\end{array}\right|
$$

For isotropic media, the off-diagonal terms in Eq. (2) vanish and

$$
r_{j}^{\mathrm{ss}}=\frac{\mu_{j} K-K_{j}}{\mu_{j} K+K_{j}}, r_{j}^{\mathrm{pp}}=\frac{\epsilon_{j} K-K_{j}}{\epsilon_{j} K+K_{j}} ; j=1,2
$$

where $K_{j}=\sqrt{\mathbf{k}_{\|}^{2}+\epsilon_{j} \mu_{j} \xi^{2} / c^{2}}$ and $\mu_{j}$ is the permeability of medium $j$.

In most cases the resulting Casimir force between the two media separated by a vacuum region is attractive. There is increased interest recently [6, 7, 8, 9] in determining whether there is a combination of media 1 and 2 capable of producing a repulsive force. There have been mainly three mechanisms to obtain repulsion for the Casimir force: (1) Dzyaloshinskii's Casimir repulsion [6]: Immersing the interacting plates of $\epsilon_{1}$ and $\epsilon_{2}$ in a fluid of $\epsilon_{3}$ and, moreover, satisfying the condition $\epsilon_{1}(i \xi)<\epsilon_{3}(i \xi)<\epsilon_{2}(i \xi)$; (2) Boyer's Casimir repulsion [7]: Based on an asymmetric setup of mainly (purely) nonmagnetic/vacuum/mainly (purely) magnetic; (3) Leonhardt's Casimir repulsion [8]: Employing a perfect lens sandwiched between the interacting plates. The possibility for a transition from an attractive to a repulsive force as the distance $d$ decreases (corresponding to a minimum of the interaction energy) leads to nanolevitations and opens up many opportunities for application, e.g., almost frictionless operation of nanomotors. Even through Capasso's group experimentally realized the repulsion, based on the theoretical prediction of Dzyaloshinskii et al. [6], this kind of system still has friction because of the existence of the liquid. Leonhardt's Casimir repulsion needs a perfect lens with simultaneously negative dielectric permittivity and magnetic permeability, which are extremely difficult to obtain at optical wavelengths. Finally, Boyer's Casimir repulsion proposal faces the essential obstacle that such nontrivial magnetic materials in the optical regime do not exist in nature, and, therefore, it relies on the nontrivial possibility of developing new artificial negative index metamaterials (NIMs).

In this letter, we examined realistic non-chiral metamaterials and we concluded they do not give a repulsive Casimir force. However, we found that chiral metamaterials are excellent candidates to realize the repulsive Casimir force. The existence of a repulsive Casimir force depends upon the strength of the chirality. We present analytical arguments that strong chirality gives a repulsive force, supported by numerical calculations.

Negative index metamaterials [10], because of their resonance magnetic response, offer more flexibility and, hence, more promise for achieving a repulsive Casimir force, based on Boyer's prediction. Indeed, in recent papers, Rosa et al. [11] found a repulsive force in a range of values of $d$ for a combination of a mainly nonmagnetic Drude-modeled silver and a magnetic NIM. This result was obtained [11] through the employment of a Lorentz type of magnetic permeability of the form $\mu(\omega)=1-\Omega^{2} /\left(\omega^{2}-\omega_{m}^{2}+i \gamma \omega\right)$. This form provides the opportunity to use an $\Omega$ large enough as to satisfy 
the condition $\mu(i \xi)>\epsilon(i \xi)$ and obtain thus Boyer's Casimir repulsion. For the reasons stated below we consider a Lorentz type frequency dependence of $\mu(\omega)$ unphysical. Instead we employed the following realistic expression for $\mu(\omega)$ :

$$
\mu(\omega)=1+\alpha-\frac{A \omega^{2}}{\omega^{2}-\omega_{m}^{2}+i \gamma_{m} \omega},
$$

where $|\alpha|$ is usually much smaller than one and $A=\alpha$ in order to satisfy the physical requirement that $\mu(\omega) \rightarrow 1$ as $\omega \rightarrow \infty$. It must be stressed that the realistic expression (4), although almost identical to the Lorentz form for $\omega$ around the resonant value $\omega_{m}$, produces radically different results than the Lorentz one as far as the Casimir attraction is concerned. As the authors of Ref. [11] have found out (and we have confirmed), expression (4) (with $\alpha=0$ and $A \neq 0$ ) combined with the form of Eq. (5) below for $\epsilon(\omega)$ does not produce repulsion. This is also true for the realistic case of $\alpha=A$.

The $\omega^{2}$ dependence of the numerator of the resonance term follows from the equivalent circuit approach [12] and from the Maxwell's equations in the low frequency regime as stated in Ref. [11]. It is confirmed by the retrieval procedure in actual SRR based and fishnet metamaterials. Of course, it is possible to have more than one resonance term in Eq. (4), but their coefficients must satisfy the relation $\alpha-\sum_{i} A_{i}=0$ to obtain the correct limiting value of $\mu(\infty)=1$. Besides cases having $\alpha=A$, we also examine the case $\alpha=0$ and $A \neq 0$ (which produces the incorrect limiting behavior, $\mu(\omega)=1-A$ as $\omega \rightarrow \infty)$. The reason for this unphysical choice is to determine the role of the $\omega=\infty$ value of $\mu(\omega)$. The most general form of the frequency-dependence of the dielectric function is the sum of the Drude term and several Lorentz-type resonance terms. If only one resonance term is kept, we have

$$
\epsilon(\omega)=1-\frac{\omega_{p l}^{2}}{\omega^{2}+i \gamma_{p l} \omega}-\frac{\omega_{e}^{2}}{\omega^{2}-\omega_{R}^{2}+i \gamma_{R} \omega} .
$$

We have calculated the Casimir force using for material 1 and material 2, $\epsilon_{1}, \mu_{1}$ and $\epsilon_{2}, \mu_{2}$, as in Eqs. (4) and (5) with several values of $A, \omega_{m}, \omega_{p l}^{2}, \omega_{e}^{2}$ (including $\omega_{p l}^{2}=0, \omega_{e}^{2} \neq$ 0 , and $\omega_{p l}^{2} \neq 0, \omega_{e}^{2}=0$ ). Among these values, we included realistic values as they were obtained by our retrieval approach in various fabricated and/or simulated NIMs. The Casimir force turned out to be attractive in all cases we calculated. See the triangle and diamond curves in Fig. 1.

Recently, a lot of experimental work on chiral metamaterials (CMMs) fabricated by planar technologies have been published [13]. For such artificial materials, the constitutive equations have the form

$$
\left(\begin{array}{l}
\mathbf{D} \\
\mathbf{B}
\end{array}\right)=\left(\begin{array}{cc}
\epsilon_{0} \epsilon & i \kappa / c_{0} \\
-i \kappa / c_{0} & \mu_{0} \mu
\end{array}\right)\left(\begin{array}{c}
\mathbf{E} \\
\mathbf{H}
\end{array}\right)
$$

where the coefficient $\kappa$ has the following frequency dependence for the chiral metamaterials [14]:

$$
\kappa(\omega)=\frac{\omega_{\kappa} \omega}{\omega^{2}-\omega_{\kappa R}^{2}+i \gamma_{\kappa} \omega},
$$

which is the same as Condon model for homogeneous chiral molecular media [15].

For such CMMs, the reflection elements can be expressed as follows, assuming the electromagnetic wave is from vacuum to chiral metamaterials [16],

$$
\begin{aligned}
& r_{j}^{\mathrm{ss}}=\frac{-\Gamma_{-}\left(\chi_{+}+\chi_{-}\right)-\left(\chi_{+} \chi_{-}-1\right)}{\Gamma_{+}\left(\chi_{+}+\chi_{-}\right)+\left(\chi_{+} \chi_{-}+1\right)}, \\
& r_{j}^{\mathrm{pp}}=\frac{\Gamma_{-}\left(\chi_{+}+\chi_{-}\right)-\left(\chi_{+} \chi_{-}-1\right)}{\Gamma_{+}\left(\chi_{+}+\chi_{-}\right)+\left(\chi_{+} \chi_{-}+1\right)}, \\
& r_{j}^{\mathrm{sp}}=\frac{i\left(\chi_{+}-\chi_{-}\right)}{\Gamma_{+}\left(\chi_{+}+\chi_{-}\right)+\left(\chi_{+} \chi_{-}+1\right)}, \\
& r_{j}^{\mathrm{ps}}=-r_{j}^{\mathrm{sp}},
\end{aligned}
$$

and

$$
\chi_{ \pm}=\frac{K_{ \pm}}{n_{ \pm} K}, \quad \Gamma_{ \pm}=\frac{\eta_{0}^{2} \pm \eta_{j}^{2}}{2 \eta_{0} \eta_{j}}
$$

where, $K_{ \pm}=\sqrt{\mathbf{k}_{\|}^{2}+n_{ \pm}^{2} \xi^{2} / c^{2}}, n_{ \pm}(i \xi)=\sqrt{\epsilon_{j}(i \xi) \mu_{j}(i \xi)} \pm$ $\kappa_{j}(i \xi), \eta_{0}=\sqrt{\mu_{0} / \epsilon_{0}}, \eta_{j}=\sqrt{\mu_{0} \mu_{j}(i \xi) / \epsilon_{0} \epsilon_{j}(i \xi)}, \epsilon_{j}(i \xi)$ and $\mu_{j}(i \xi)$ are the relative permittivity and permeability of the plate $j$, respectively, and $\kappa_{j}(i \xi)$ is the chirality coefficient. Although $n_{ \pm}$are complex, the reflection elements, r's, are still purely real because $\chi_{+}=\chi_{-}^{*}$.

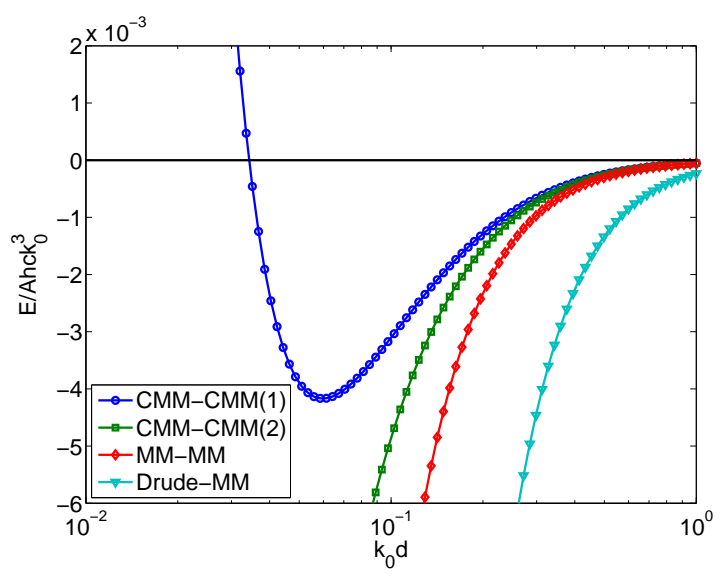

FIG. 1: (Color online) Casimir interaction energy per unit area $E / A$ (in units of $h c k_{0}^{3}$ ) versus $k_{0} d ; k_{0}=\omega_{R} / c$. The triangle curve corresponds to $\alpha=A=0.001, \kappa=0$ (no chirality), $\omega_{m}=$ $\omega_{R}, \gamma_{m}=\gamma_{R}=0.05 \omega_{R}, \omega_{p l}=0, \omega_{e}=\omega_{R}$ for material 1, while $\alpha=A=0, \omega_{p l}=10 \omega_{R}, \gamma_{p l}=0.05 \omega_{p l}, \omega_{e}=0$ for material 2. The diamond curve is the case with $\alpha=A=0.001, \kappa=0, \omega_{m}=$ $\omega_{R}, \gamma_{m}=\gamma_{R}=0.05 \omega_{R}, \omega_{p l}=0, \omega_{e}=\omega_{R}$. The squares curve is the case with $\alpha=A=0.001, \omega_{\kappa 1}=\omega_{\kappa 2}=0.6 \omega_{R}, \omega_{m}=$ $\omega_{\kappa R}=\omega_{R}, \gamma_{m}=\gamma_{\kappa}=\gamma_{R}=0.05 \omega_{R}, \omega_{p l}=0, \omega_{e}=\omega_{R}$. Finally, the circle curve shows repulsion for $k_{0} d<0.0586$ and a stable equilibrium point at $k_{0} d=0.0586$; the parameters are the same as for the square curve except for $\omega_{\kappa 1}=\omega_{\kappa 2}=0.7 \omega_{R}$.

Here, we consider first a special setup with two identical chiral metamaterial plates with the following parameters: $\epsilon_{1}=\epsilon_{2}=\epsilon ; \mu_{1}=\mu_{2}=\mu ; \kappa_{1}=\kappa_{2}=\kappa$. We suspect that the 
chirality coefficient, $\kappa$, may provide sufficient new freedom to drive the force to negative values (i.e., repulsive) at least for some range of values of $d$. From Eq. [1] it follows Ref. [11] that a negative value of the Casimir force is favored by making the quantity $I \equiv \operatorname{Tr}\left[\mathbf{D}^{-1}(1-\mathbf{D})\right]$ as negative as possible over as broad a range as possible of the parameters and the integration variables. This quantity, $I$, has the same sign as the quantity $F$ given below:

$F=\frac{\left(r_{s s}^{2}+r_{p p}^{2}-2 r_{s p}^{2}\right) e^{-2 K d}-2\left(r_{s p}^{2}+r_{s s} r_{p p}\right)^{2} e^{-4 K d}}{1-\left(r_{s s}^{2}+r_{p p}^{2}-2 r_{s p}^{2}\right) e^{-2 K d}+\left(r_{s p}^{2}+r_{s s} r_{p p}\right)^{2} e^{-4 K d}}$

Because $r_{s p}$ is purely real as shown in Eq. (8), it is clear from Eq. (9) that the chirality by introducing the off-diagonal quantity $r_{s p}$ provides the possibility, for large enough $r_{s p}$, to make the numerator in Eq. (9) negative, while keeping the denominator positive. Thus, the chirality, if strong enough, is expected to lead to a repulsive Casimir force. This expectation is confirmed by the numerical evaluation of the interaction energy per unit area as shown in Fig. 1 Indeed, for large enough chirality parameter, $\omega_{\kappa 1}=\omega_{\kappa 2}=0.7 \omega_{R}$, we have a very interesting situation of an attractive force in the range $d>d_{0}$ (where in the present case $d_{0}=0.0586 \mathrm{c} / \omega_{R}$ ) and a repulsive case for $d<d_{0}$. Thus, a stable equilibrium distance emerges, $d=d_{0}$, reminiscent of the bond length in a diatomic molecule. There is a critical value of $\omega_{\kappa}, \omega_{\kappa}=\omega_{\kappa}^{c}$, such that for $\omega_{\kappa}<\omega_{\kappa}^{c}$ there is no repulsive regime for any value of $d$, while for $\omega_{\kappa}>\omega_{\kappa}^{c}$, there is a distance $d_{0}$, a function of $\omega_{\kappa}, d_{0}\left(\omega_{\kappa}\right)$, such for $d<d_{0}\left(\omega_{\kappa}\right)$ the force is repulsive. For the numerical values used in our present case, the critical value of $\omega_{\kappa}^{c}$ is equal to $\omega_{\kappa}^{c}=0.612 \omega_{R}$ for $\alpha(=A)=0$. As shown in Fig. 2(a), the critical value $\omega_{\kappa}^{c}$ is a function of the $\alpha$ with its minimum value $\omega_{\kappa}^{c}=0.607$ obtained for $\alpha \simeq-0.09$. Furthermore, the relation $d_{0}$ versus $\omega_{\kappa}$ (for $\omega_{\kappa}>\omega_{\kappa}^{c}$ ) is an increasing almost linear function of $\omega_{\kappa}$, as shown in Fig. 2(b).
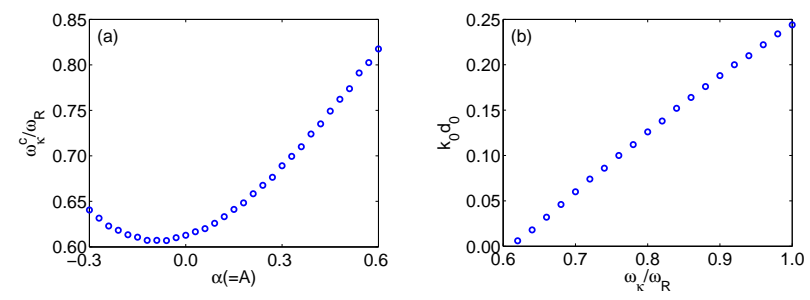

FIG. 2: (Color online) (a) The critical value of chirality $\omega_{\kappa}^{c}$ versus $\alpha(=A)$, for two identical CMM plates. (b) The equilibrium distance $k_{0} d_{0}$ versus $\omega_{\kappa}$ for $\alpha=A=10^{-3}$. For $\omega_{\kappa}>\omega_{\kappa}^{c}=0.612 \omega_{R}$, the value of the equilibrium distance $k_{0} d_{0}$ corresponds to the minimum of the energy as shown by the open circle curve in Fig. 1 .

The question raised by the present novel approach to a possible repulsive Casimir force is whether real chiral metamaterials can be fabricated with $\omega_{\kappa}$ larger than the critical one $\omega_{\kappa}^{c}$. Our own chiral metamaterial presented in Ref. [13] has an $\omega_{\kappa} \simeq 0.3 \omega_{\kappa}^{c}$. However, this metamaterial was designed and fabricated before the critical importance of chirality for sta- ble Casimir nanolevitation was even suspected; thus, there is room for new designs to raise the value of $\omega_{\kappa}$ possibly above the critical value. We are currently working on this theme. We don't know whether or not general physical considerations restrict the size of the chirality factor $\omega_{\kappa}$ and thus we cannot be sure whether the critical value of $\omega_{\kappa}^{c}$ is reachable. Models based on a single loop (see the books of Lindell et al. [16] and Serdyukov et al. [17]) produce a relation between the electric, $\alpha_{e e}$, the magnetic, $\alpha_{m m}$, and the cross polarizabilities, $\alpha_{e m}, \alpha_{m e}: \alpha_{e e} \alpha_{m m}=\alpha_{e m} \alpha_{m e}$. This relation, valid when $\omega_{m}=\omega_{R}=\omega_{\kappa R}, \gamma_{m}=\gamma_{R}=\gamma_{\kappa}$ and $\omega_{p l}=\alpha=0, A \neq 0$, shows that the critical value $\omega_{\kappa}^{c}$ is almost reachable under the optimum condition $A \rightarrow \omega_{e}^{2} / \omega_{R}^{2}$.

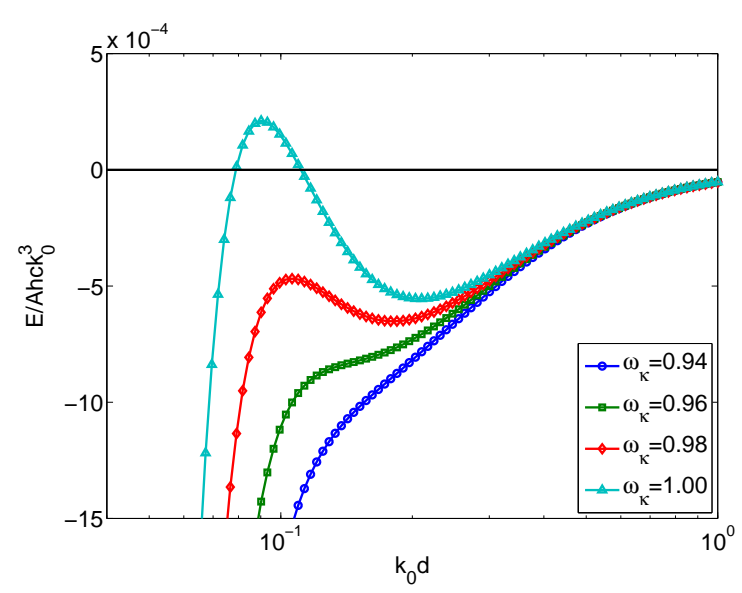

FIG. 3: (Color online) Casimir interaction energy per unit area E/A (in units of $h c k_{0}^{3}$ ) versus $k_{0} d$ of the two identical CMM plates configuration for different chiral strengths $\omega_{\kappa}$ 's. $\alpha=0, A=0.2, \omega_{p l}=$ $0, \omega_{m}=\omega_{\kappa R}=\omega_{R}, \gamma_{e}=\gamma_{m}=\gamma_{\kappa}=0.05 \omega_{R}, \omega_{e}=\omega_{R}$.

In Fig. 3 we present results for the energy per unit area $E / A$ versus the dimensionless distance $k_{0} d$ for chiral metamaterials with $\mu(\omega)$ given by Eq. (4) with $\alpha=0$ and $A=0.2$. We repeat here this choice violates the physical requirement of $\mu(\omega) \rightarrow 1$ as $\omega \rightarrow \infty$. Nevertheless, we present these results here in order to show that unphysical frequency dependence of the response functions may produce the resulting behavior which is qualitatively different from that presented in Fig. 11in the sense that now two equilibrium points, $d_{1}$ and $d_{2}\left(d_{1}<d_{2}\right)$, may appear, the first is unstable equilibrium and the second is stable equilibrium. Furthermore, one cannot exclude the possibility that a more complicated $\mu(\omega)$ satisfying the condition $\mu(\infty)=1$ and producing results as those in Fig. 3 may exist. In spite of this unphysical behavior of $\mu(\infty)(\mu(\infty)=0.8$ instead of $\mu(\infty)=1)$, one expects to produce no repulsive force if regular metamaterials (with no chirality) are employed. The reason is that both of the interacting plates are mainly nonmagnetic with $\mu(i \xi)<1<\epsilon(i \xi)$ at all frequencies. For a not so large chirality $\left(\omega_{\kappa}=0.94\right.$ (circles)), one can easily see from Fig. 3 there is only an attractive Casimir force for all distances. However, as chirality increases, $\left(\omega_{\kappa}=0.96\right.$ (squares)), the energy tries to 
develop a minimum, and the Casimir force corresponding to the slope of the energy becomes smaller. At $\omega_{\kappa}=0.98$ (diamonds), there is a minimum at $k_{0} d_{2} \simeq 0.21$ and a maximum at $k_{0} d_{1} \simeq 0.09$; the peak value of the energy is less than zero, the energy value at $k_{0} d=\infty$. If chirality increases further, $\omega_{\kappa}=1.00$ (triangles), the sign of the energy is reversed and becomes positive in a certain range. This is an interesting case that gives a repulsive Casimir force within a range of distances between $d_{1}$ and $d_{2}$. It forms a potential barrier to block the two interacting plates sticking to each other. Similar results to those in Fig. 3 were also obtained for the case where we used $\epsilon(\omega)=2-\omega_{e}^{2} /\left(\omega^{2}-\omega_{e}^{2}+i \gamma \omega\right)$. This frequency-dependence is obtained experimentally [10] for realistic metamaterials, but only close to the resonance behavior; such a dependence extended to $\omega \rightarrow \infty$ violates the condition of $\epsilon(\omega) \rightarrow 1$ as $\omega \rightarrow \infty$.

In discussing these results we must keep in mind that for $k_{0} d \ll 1$ the main contribution to the integral in Eq. (1) comes from large $\xi$ and $k_{\|}$values with the ratio $k_{\|} / \xi \gg 1$, as argued by Landau et al. [18] and confirmed by our numerical calculations. Under these conditions $k \simeq k_{\|}$and the integrand in Eq. (1) takes the form $f\left(\xi, e^{-2 k_{\|} d}\right)$. By setting $x=2 k_{\|} d$, it follows immediately from Eq. (1) that $E(d) / A \propto d^{-2}$ and $F(d) / A \propto d^{-3}$; the contribution of the chiral term to $f\left(\xi, e^{-x}\right)$ is negative and, thus, for large enough chirality the force in the $d \rightarrow 0$ limit becomes repulsive. On the other hand, in the opposite limit $d \rightarrow \infty$, because of the factor $e^{-2 K d}$, the main contribution to the integral comes from the range $0 \leq \xi \lesssim(c / d)$ and $0 \lesssim k_{\|} \lesssim\left(d^{-1}\right)$, where the integrand tends to a constant corresponding to the $\xi=0$ values of $\epsilon(0)>1, \mu(0) \simeq 1$, and $\kappa(0)=0$. Thus in this $d \rightarrow \infty$ limit $E(d) / A \propto d^{-3}$ and $F(d) / A \propto d^{-4}$ and the force is always repulsive, since essentially only $\epsilon(0)$ matters. This analysis shows that it is crucial to employ the correct limiting values of $\epsilon(i \xi), \mu(i \xi), \kappa(i \xi)$ as $\xi \rightarrow \infty$ and $\xi \rightarrow 0$, since these values determine the behavior of $E(d) / A$ in the limit $d \rightarrow 0$ and $d \rightarrow \infty$ respectively.

In this work we have extended the Lifshitz theory to calculate the Casimir force by including chirality terms for the first time. We have shown that the chirality, if strong enough, is of critical importance in producing nanolevitations under realistic frequency-dependence and correct limiting values of $\epsilon(\omega)$ and $\mu(\omega)$. Note, the previous calculations claiming repulsive Casimir force between metamaterials separated by vacuum have been achieved at the expense of nonrealistic frequencydependence and/or limiting values of $\epsilon(\omega)$ and $\mu(\omega)$. Thus, chiral metamaterials might possibly be the main candidates to achieve experimentally the goal of Casimir repulsion, which might open up many opportunities for application.

Work at Ames Laboratory was supported by the Department of Energy (Basic Energy Sciences) under contract No. DE-AC02-07CH11358. This work was partially supported by the European Community FET project PHOME (contract No. 213390), US Department of Commerce NIST 70NANB7H6138 and the US Air Force grants. The author
Rongkuo Zhao specially acknowledges the China Scholarship Council (CSC).

[1] H. B. G. Casimir, Proc. Kon. Nederl. Akad. Wet. 51, 793 (1948).

[2] E. M. Lifshitz, Sov. Phys. JETP 2, 73 (1956).

[3] V. A. Parsegian and G. H. Weiss, J. Adhes. 3, 259 (1972); Y. S. Barash, et al., Radiofiz., 12, 1637 (1978) [Radiophysics and Quantum Electronics, 21, 1138 (1978)]; T. G. Philbin and U. Leonhardt, Phys. Rev. A 78, 042107 (2008), and references therein.

[4] A. Lambrecht, P. A Maia Neto, and S. Reynaud, New J. Phys. 8, 243 (2006), and references therein.

[5] A conceptually (but not calculationally) simple way to obtain Eqs. (1) and 23 is to calculate the surface electromagnetic eigenmodes $\omega\left(\mathbf{k}_{\|}\right)$of this system and then to evaluate their zero point energy, $\sum_{i} \int d \mathbf{k}_{\|} \frac{1}{2} \hbar \omega_{i}\left(\mathbf{k}_{\|}\right)$.

[6] J. N. Munday, F. Capasso, and V. A. Parsegian, Nature 457, 170 (2009); F. Capasso et al., IEEE J. Sel. Top. Quantum Electron. 13,400 (2007); I. E. Dzyaloshinskii, E. M. Lifshitz, and L. P. Pitaevskii, Adv. Phys. 10, 165 (1961).

[7] T. H. Boyer, Phys. Rev. A 9, 2078 (1974); O. Kenneth et al., Phys. Rev. Lett. 89, 033001 (2002).

[8] U. Leonhardt and T. G. Philbin, New J. Phys. 9, 254 (2007).

[9] F. Intravaia and A. Lambrecht, Phys. Rev. Lett. 94, 110404 (2005); Phys. Rev. A 76033820 (2007).

[10] S. Linden et al., Science 306, 1351 (2004); C. M. Soukoulis, S. Linden, and M. Wegener, Science 315, 5808 (2007); C. Enkrich et al., Phys. Rev. Lett. 95, 203901 (2005).

[11] F. S. S. Rosa, D. A. R. Dalvit, and P. W. Milonni, Phys. Rev. Lett. 100, 183602 (2008), Phys. Rev. A 78, 032117 (2008), and references therein; G. Deng et al., Phys. Rev. A 78, 062111 (2008).

[12] J. B. Pendry et al., IEEE Trans. Microwave Theory Tech. 47, 2075 (1999) M. Gorkunov et al., Eur. Phys. J. B 28, 263 (2002).

[13] E. Plum et al., Phys. Rev. B 79, 035407 (2009); S. Zhang et al., Phys. Rev. Lett. 102, 023901 (2009); J. Zhou et al., Phys. Rev. B 79, 121104(R) (2009); B. Wang et al., Appl. Phys. Lett. 94, 151112 (2009).

[14] We have proven, based on the equivalent circuit approach, that $\kappa$ should obey this form with a linear $\omega$ dependence of the numerator and our fitting results using this formula agree with our simulations and experiments very well. Finally, the linear $\omega$ dependence for low frequencies is a general feature of natural optically active materials (see L.D. Landau et al., Electrodynamics of Continuous Media (2nd ed., Pergamon Press, Oxford, 1984), $\S 104$, p.362-367). Here, we should note that there is an $\omega$ in the numerator, therefore, $\kappa(i \xi)$ is pure imaginary.

[15] E. U. Condon, Rev. Mod. Phys. 9, 432-457 (1937).

[16] See, for instance, A. Lakhtakia et al., J. Opt. Soc. Am. A 7, 1654 (1990); I. V. Lindell et al., Electromagnetic Waves in Chiral and Bi-Isotropic Media (Artech House, Boston · London, 1994).

[17] A. Serdyukov et al., Electromagnetics of Bi-anisotropic Materials: Theory and Applications (Gordon and Breach Science Publishers, Amsterdam, 2001).

[18] E.M. Lifshitz and L.P. Pitaevskii, Statistical Physics (3rd ed., Pergmon Press, Oxford, 1980), Part 2, §80-82. 